\title{
Genome and karyotype evolution after whole genome duplication in free-living flatworms of the genus Macrostomum
}

\author{
Kira Zadesenets \\ ICG SB RAS, Novosibirsk, Russia \\ kira_z@bionet.nsc.ru \\ Eugene Berezikov \\ ERIBA, Groningen, The Netherlands \\ ICG SB RAS, Novosibirsk, Russia \\ e.berezikov@umcg.nl
}

\author{
Nikita Ershov \\ ICG SB RAS, Novosibirsk, Russia \\ nikotinmail@mail.ru \\ Lukas Schärer \\ Evolutionary Biology, Zoological \\ Institute, University of Basel, Basel, \\ Switzerland \\ lukas.scharer@unibas.ch
}

\author{
Dmitry Oshchepkov \\ ICG SB RAS, Novosibirsk, Russia \\ diman@bionet.nsc.ru \\ Nikolay B. Rubtsov \\ ICG SB RAS, Novosibirsk, Russia \\ rubt@bionet.nsc.ru
}

\begin{abstract}
Whole genome duplication (WGD) is a largescale evolutionary transformation that took place in genome evolution in many taxa of existing animal species. However, the mechanisms underlying the early stages of genome evolution after a WGD event in animals has remained unclear. The study of genome organization of neopolyploid species may shed light on the processes of genome reorganization leading to its re-diploidization after a recent round of WGD.
\end{abstract}

Keywords - whole genome duplication, hidden polyploidy, karyotype instability, genome evolution, macrostomids, flatworms

\section{Motivation and aim}

\section{Motivation and Aim}

Earlier we uncovered a group of free-living flatworms in which genomes have likely undergone a recent WGD. We found out that karyotype instability was linked to hidden polyploidy in both species $M$. lignano $(2 \mathrm{n}=8)$ and its sibling species $M$. janickei $(2 \mathrm{n}=10)[1,2]$. Additionally we studied other species of the genus Macrostomum and revealed a new species (further called $M$. mirumnovem) with a highly unstable karyotype. We studied the detailed karyotype and genome organization in three Macrostomum species.

\section{Methods}

To describe karyotype diversity in the Macrostomum species (M. lignano, M. janickei, M. mirumnovem) the routine single-worm karyotyping was performed. The detailed cytogenetic analyses were made using a set of different DNA probes (microdissected region- and chromosome specific DNA probes, DNA repeats, unique DNA fragments). Microdissected DNA probes (specific to regions and whole chromosomes) were generated for the $M$. lignano and M. mirumnovem chromosomes. The generated chromosome-specific DNA probes for the M. lignano chromosomes were sequenced and the obtained NGS data were used for following bioinformatics analyses using the existing genome assemblies for the M. lignano genome.

\section{Results}

The detailed cytogenetic analyses using a set of different DNA probes (microdissected region- and chromosome specific DNA probes, DNA repeats, unique DNA fragments) revealed the peculiarities of karyotype and genome organization in three Macrostomum species with unstable karyotypes. Similar to M. lignano and M. janickei, our findings suggest that $M$. mirumnovem arose via whole genome duplication (WGD) followed by considerable reshuffling of its chromosomes. The combined approach allowed us to explore the peculiarity of the M. lignano genome organization, the strategy included bioinformatics analysis of the existed genome assemblies of $M$. lignano and NGS data for its separate chromosomes. Based on the obtained results, we suggest possible evolutionary scenarios for the emergence and reorganization of the karyotypes and genomes of the post-WGD Macrostomum species.

\section{Acknowledgment}

This study is supported by grant of the Russian Science Foundation (RSF) (project No. 19-14-00211).

\section{References}

[1] Zadesenets K.S. et al. (2016) Evidence for karyotype polymorphism in the free-living flatworm, Macrostomum lignano, a model organism for evolutionary and developmental biology. PLoS One. 11(10): e0164915.

[2] Zadesenets K.S., Schärer L., Rubtsov N.B. (2017) New insights into the karyotype evolution of the free-living flatworm Macrostomum lignano (Platyhelminthes, Turbellaria). Sci. Rep. 7: 6066. 